OnLine Journal of Biological Sciences 12 (2): 44-53, 2012

ISSN 1608-4217

(C) 2012 M. Mishra et al., This open access article is distributed under a Creative Commons Attribution

(CC-BY) 3.0 license

\title{
Developing a System for Antibacterial Treatment of Dental Caries using Culture Based Approach
}

\author{
${ }^{1}$ Mishra, M., ${ }^{1}$ S. Ghosh, ${ }^{1}$ L.E. Alex, \\ ${ }^{2}$ I. Mukherjee, ${ }^{3}$ T.P. Sinha and ${ }^{1}$ S. Ray Chaudhuri \\ ${ }^{1}$ Department of Biotechnology, \\ ${ }^{2}$ Department of Natural Sciences, \\ West Bengal University of Technology, \\ BF-142, Sector-1, Salt Lake, Kolkata-700064, West Bengal, India \\ ${ }^{3}$ Department of Physics, Bose Institute, 93/1, A.P.C. Road, Kolkata-700009, India
}

\begin{abstract}
Problem statement: Dental caries is a chronic disease worldwide, caused mainly by microbes. The orthograde approaches still remain the only option of treatment. Lack of rapid antibiotic sensitivity determination of the dental pathogen is a rate limiting step in the field of Dental Microbiology. Approach: The culture dependent analysis was used for the study of oral microflora from five healthy individuals as well as three caries infected buccal cavity (one with pit and fissure caries and two with smooth surface caries). Results: The study revealed $10^{1}-10^{6}$ times higher bacterial count in healthy buccal cavity. The molecular community analysis mostly revealed Klebsiella sp, Staphylococcus sp, Bacillius sp., Streptococcus sp. and Granulicatella sp in caries infected samples. Microbial isolates from surface caries of one of the subjects (DS5 and DS6) showed resistance to most of the drugs except Polymyxin B, while Ampicillin resistance was common for all. Most of the consortia from both the healthy and infected individuals' revealed presence of plasmids. Two step cultivation based antibiotic sensitivity detection gave consistent result within $18-20 \mathrm{~h}$ as opposed to the conventional 48-72 h. A model for oral application of antibiotic to tooth surface was worked out to inhibit demineralization. Conclusion/Recommendation: This study reports a rapid antibiotic sensitivity detection system for selection of treatment regime. It also proposes a model antibiotic applicator for treatment of surface caries. Detection of antibiotic sensitivity before administration of antibiotic can prevent misuse of antibiotic therapy leading to enhancement of multi drug resistance. The therapy conducted on the basis of the sensitivity data can prevent the demineralization and escaping of the isolates into the blood stream during tooth extraction.
\end{abstract}

Key words: Dental microflora, surface caries, pit and fissure caries, demineralization, Shannon diversity, Mueller Hinton Agar (MHA), Luria Bertani (LB)

\section{INTRODUCTION}

Tooth decay otherwise known as dental caries is one of the most common chronic diseases prevailing in the human population, its epidemiology rate being stated as five times greater than that of asthma (Houte, 1994). This disease can be defined as an irreversible infection of teeth and is usually considered as bacterial in origin (Shivkumar et al., 2009). It is basically a dynamic process taking place in the microbial deposits on the teeth otherwise known as plaque. The acidogenic bacteria inhabiting the microbial biofilm utilize the fermentable carbohydrates and as a consequence lower the $\mathrm{pH}$ of the surrounding area. This acidic condition causes demineralization of the dentin and cementum and even degrades the organic matter of the teeth (Hardie, 1992; Liljemark and Bloomquist, 1996; Houte, 1994). Bacterial population can even cause proteolytic destruction of the collagen matrix eventually leading to irreversible demineralization of dentine.

Human oral cavity can be considered as a complex ecosystem with diverse acid tolerant and acid producing microbiota. Extensive reports based on culture dependent and independent approaches give an account for existence of 200-300 species in human dental plaque (Islam et al., 2007). Among the myriad of population in the oral cavity, major fraction of about $70 \%$ in reported to be noncultivable (Aas et al., 2005).

Corresponding Author: S. Ray Chaudhuri, Department of Biotechnology, West Bengal University of Technology, BF-142, Sector-1, Salt Lake, Kolkata-700064, West Bengal, India 
The availability of nutrients and moisture, favourable temperature and occurrence of adherence surface inside the oral cavity creates a compatible environment for development of microbial biofilm. The proteins and glycoprotein in the host's mouth prepare a conditioning film for plaque initiation (Islam et al., 2007; Houte, 1994).

A number of investigations link Streptococcus mutans, an acidogenic bacterium to the development of caries (Aas et al., 2008; Islam et al., 2007; Becker et al., 2002; Preza et al., 2008). Apart from their fermentative ability, these bacteria are known to produce insoluble polysaccharides like dextran which enhances the biofilm development. Along the progressive stages of the caries the bacterial profile changes and species of genera Vellionella, Lactobacillus, Bifidobacterium, Propionibacterium, Actinomyces, Enterococcus, Atopobium, Selenomonas, Prevotella and Pseudoramibacter take up a major role. Species of Lactobacillus and Vellionella are weakly adherent and thus depend on $S$ mutans for caries initiation (Gross et al., 2010). Moreover the predominance of Lactobacillius in the secondary dentines is due to the production of antimicrobials which inhibits the growth of $S$ mutans (Houte, 1994). Similarly Velionella sp. acts as a sink for the lactic acid produced by the preliminary inhabitants to generate weaker acids like propionic and acetic acid. Thereby it stimulates the glycolysis process in the lactic acid producers like Streptococcus and Lactobacillus (Becker et al., 2002). The incidence of different microbial groups is linked to the stages of infection and is explained by ecologic disruption model of caries pathobiology (Li et al., 2005). Thus, microbial screening of healthy and infected teeth is essential to understand the potent ecological role of the different species associated with caries. The dangers of this disease can be linked to the various systemic disorders such as infective endocarditis, bacteremic seeding of heart valves and even increased risk of coronary heart disease and cerebrovascular events. Excessive bacterial load in the oral cavity may even lead to gingivitis, periodontics, periimplantis (Cate, 2009; Marsh, 2003; Nakano et al., 2009). These oral bacteria can enter the blood stream during dental procedures such as tooth extraction, periodontal surgery, scaling and root planning or even regular brushing, flushing or even during chewing of food. By far the most widely used therapeutic method is fluoride based since its application prevents dissolution and allows reprecipitation of enamel and dentin. But regular use of fluoride can have negative impacts like fluorosis and other toxicological effects. Another conventional approach is the application of antimicrobials with an aim to eliminate the arch cariogenic species. Chlorhexidine digluconate (CHX) is a commonly reported drug which eliminates or suppresses $S$ mutans (Islam et al., 2007). Other commonly used antimicrobials are Amoxicillin, Kanamycin, Tetracycline, Cefepime sulbactum in combination (Srivastava and Chaudhury, 2011). But again the rampant use of antimicrobials leads to sharp rise in antibiotic resistance of microbes which stands as a hindrance in disease prevention and treatment (Ready et al., 2002; Roberts, 1998; Srivastava and Chaudhury, 2011). In the light of the above consequences, significant effort is devoted towards understanding the caries etiology and designing alternatives for prevention and therapeutic intervention.

The purpose of this present study in three folds (a) a culture based microbial screening of healthy and infected teeth in adults, (b) to perform a rapid method of antibiotic sensitivity detection to facilitate the diagnostic process, (c) development of an effective treatment method. This study would lead to a detailed understanding of caries pathobiology and contribute to improved prevention and treatment of this significant health problem.

\section{MATERIALS AND METHODS}

Subject selection: Three adult subjects were selected who ranged in age from 35-70 years. The first Subject (S1) had deep pit and fissure caries, the second Subject (S2) had severe surface caries whereas the third Subject (S3) was affected with severe rampant and surface caries. None of the subjects were exposed to any immediate antibiotic treatment before being selected for microbial screening. The healthy control group consisted of five subjects (HS1-HS5) within the age range of 22-28 years. The control subjects did not receive any antibiotic treatment in the recent past before being enrolled in this study.

Sampling for culture dependent screening: A jet of sterile water was dispensed from a $2 \mathrm{~mL}$ syringe in order to wash the tooth surface. The water was sucked back from the tooth surface and stored in a microfuge tube for further analysis. $50 \mu \mathrm{L}$ of the collected sample was evenly spread on Luria Bertani (LB) agar (1\% Tryptone, $0.5 \%$ Yeast extract, $0.5 \% \mathrm{NaCl}, 1.5 \%$ agar, $\mathrm{pH}$ 7-7.5) and was incubated at $37^{\circ} \mathrm{C}$ for overnight. Sterile water was used as negative control for each set of experiment in case of both healthy and infected teeth. The preliminary analysis was based on viable cell count in terms of $\mathrm{CFU} / \mathrm{mL}$ and the basic colony morphology. 


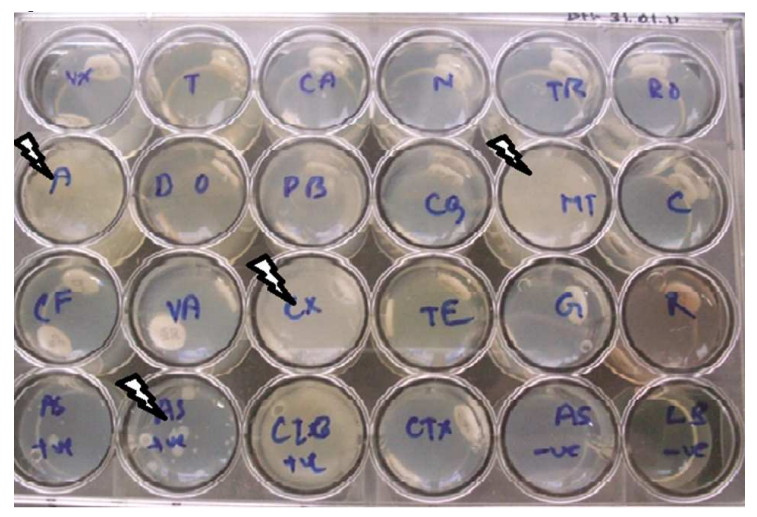

Fig. 1: Picture of a tissue culture plate depicting the arrangement of the antibiotic sensitivity kit that could be used for clinical and environmental sample screening. The arrow indicates wells with growth. The same kit was also used for antibiotic sensitivity detection of contaminated blood, sputum and urine

Two of the consortia, one obtained from the buccal cavity of S1 and the second from S2 were further selected for obtaining pure isolates by following the pure culture technique as reported earlier (Adarsh et al., 2007).

Molecular characterization of consortia: The molecular characterization of the different consortia as well as that of the pure isolates obtained from the infected buccal cavities was done on the basis of $16 \mathrm{~S}$ rDNA sequence analysis. The detailed steps of molecular characterization were followed as reported by Chaudhuri and Thakur (2006). The sequences were analyzed by BLAST against GenBank in order to depict the identity of the closest neighbours and thereafter the novel sequences were submitted to GenBank. The taxonomic assignments were made by the Neighbor Joining method.

Statistical analysis: To analyze the species richness and evenness, Shannon Diversity Index (H) and Equitability Index were calculated for each consortium by following the principle reported by Gafan et al. (2005) and Nasipuri et al. (2010).

Antibiotic sensitivity assay: The antibiotic sensitivity of the various consortia as well as that of the pure isolates was checked against 18 different antibiotics by disc diffusion assay on Mueller Hinton Agar (MHA) media (Himedia M173). The different antibiotics used and the detailed procedure for sensitivity assay was followed as reported by Adarsh et al. (2007).
Screening for the presence of plasmids: Since the consortia as well as the pure isolates demonstrated antibiotic resistance, an attempt was made to screen for the presence of plasmids in them as an indirect method to understand the molecular mechanism of antibiotic resistance. The plasmid isolation, transformation into ampicillin sensitive hosts and finally screening of positive transformants were done by following the procedure reported by Chaudhuri and Thakur (2006).

Development of a rapid antibiotic sensitivity detection kit: Since the standard antibiotic sensitivity assay for biological sample was a time consuming process, a modified method was designed to reduce the detection time of the antibiotic sensitivity.

A jet of $20 \mu \mathrm{L}$ sterile water was used to wash the surface of the infected tooth. Thereafter a swab stick was used to collect swab samples from the washed tooth surface. The swab stick was transferred to $3 \mathrm{~mL}$ of AS broth (1\% Tryptone, $0.5 \% \mathrm{NaCl}, \mathrm{pH} 7.0)$, mixed properly by vortex and incubated at $37^{\circ} \mathrm{C}$ for $2 \mathrm{~h}$ under shaking condition at $150 \mathrm{rpm}$ (Shaker, Lab companion, SI 300R). This initial culture was used as innoculum for the second step of detection on culture plates and therefore the procedure is called the two step cultivation technique.

In the second step of the analysis, a 24 well tissue culture plate (Cell star cat no.662160) was used with 2 wells containing $2 \mathrm{~mL} \mathrm{LB}$ agar each while the rest 22 wells were filled with $2 \mathrm{~mL}$ of AS agar (1\% Tryptone, $0.5 \% \mathrm{NaCl}, 1.5 \%$ Agar). The wells were then marked according to the eighteen different antibiotics. For each set, two of the AS agar containing wells were considered as positive controls and one AS agar containing well was labelled as negative control. The two LB agar containing wells were used as positive and negative control respectively. In the wells marked for respective antibiotic, two discs were aseptically inserted into the corresponding wells and the insertion points were sealed with top agar (Fig. 1). Then $20 \mu \mathrm{L}$ from incubated culture (initial culture) was spread uniformly on agar by swirling. The sensitivity and resistance towards a particular antibiotic was determined in terms of presence or absence of microbial growth after an incubation period of $16 \mathrm{~h}$. Thus the total time for detection remains as $18 \mathrm{~h}$.

Assessment of local antibiotic application on healthy and infected teeth: Goat tooth was selected as a model for the study of antibiotic effect on healthy and infected teeth. Three sets of front jaw teeth were collected from a healthy buccal cavity. With an objective of eliminating any preexisting microbe, the teeth sets were 
initially washed with distilled water for $30 \mathrm{~min}$ followed by $70 \%$ ethanol wash for $1 \mathrm{~h}$. Thereafter they were exposed to UV irradiation for $1 \mathrm{~h}$ to kill the surface adherent microbes. The teeth sets were placed on petriplates with aid of plasticin like material and thereafter supplied with simulated saliva (Ghosh et al., 2009) and drops of $1 \mathrm{M}$ sucrose.

Among the three sets of teeth, one was kept as the control i.e., healthy teeth. The second set of tooth was treated once with a well characterized strain of Staphylococcus haemolyticus (Nandy et al., 2007; 2009; Chaudhuri et al., 2008) which is a known cariogenic species. The third set was infected with the above mentioned bacterial isolate and thereafter treated with an antibiotic combination of Polymixin B and Neomycin (commercially available in the trade name of Neosporin). The selection of antibiotics was based on the antibiotic sensitivity of this particular Staphylococcus strain (Nandy et al., 2007; 2009; Chaudhuri et al., 2008). Each set was then placed in a 2 liter plastic vessel half filled with water and maintained at $37^{\circ} \mathrm{C}$ to simulate the condition in the buccal cavity. The treatment with sucrose as well as antibiotic was given to the respective teeth for 21 days. After the incubation period, the tooth samples were washed sequentially with distilled water, $100 \%$ ethanol, and chloroform for removing any adherent material from the surface. Scanning Electron Microscopy (FEI QUANTA 200 MARK2) was done to analyze the morphological alterations of the teeth surface with and without antibiotic treatment following silver coating.

\section{RESULTS}

Isolation and enumeration of dental flora from healthy and infected teeth surfaces: Six consortia were obtained from infected as well as non infected dentine surface from the bucccal cavities of infected subjects (S1, S2 and S3), their nomenclature and the details of the isolation source being provided in Table 1 . The viable cell count $(\mathrm{CFU} / \mathrm{mL})$ was derived for each consortium obtained from infected (S1-S3) as well as healthy subjects i.e. HS1-HS5 (Table 1).

Three pure bacterial isolates were obtained. One was from consortia DS3 which was from the non infected tooth of S1 while two others were from DS4 which was from the infected tooth of sample S2.

Molecular characterization of the consortia: The molecular analysis revealed the predominance of Klebsiella sp. in consortia DS1, DS2 and DS3, Staphylococcus sp. in consortium DS4, Bacillius and
Staphylococcus sp. in consortium DS5 while occurrence of Streptococcus sp., Granulicatella sp. as well as an unknown bacterium in consortium DS6. The representative phylogenetic trees derived for consortia DS1-DS6 showing the relationship of species and phylotypes were depicted in Fig. 2a-f. The pure isolates SRC_DSa and SRC_DSb demonstrated closest identity with the unknown bacterium. The sequences being novel were submitted to GenBank, the accession numbers were provided in Table 2. Though 25 clones were screened from each consortium but the diversity obtained was very poor and hence rarefaction curve was not attempted.

The Shannon Diversity Index $(\mathrm{H})$ as well as the Equitability Index for the consortia DS1 to DS6 was mentioned in Table 2. The values were extremely low indicating low diversity as well as the uneven distribution of members in the community except for DS4 which had a nearly even distribution of population.

Antibiotic sensitivity assay: The sensitivity to 18 different antibiotics were checked for different consortia obtained from healthy as well as infected buccal cavities and also the pure bacterial isolates, as reported in Table 3. All consortia except DS4 were resistant to Metronidazole as well as Ampicillin. Rifampicin sensitivity was demonstrated by DS4 and pure isolate SRC_DSb, while both demonstrated intermediate response towards Cloxacillin. Polymyxin B was effective for all the consortia from infected buccal cavities while most of the consortia from healthy subjects were resistant to it. The antibiotic sensitivity profile is essential to understand the caries ecology and also determine the targets for therapeutic intervention.

Table 1: Details of the isolation source of the different consortia obtained from the buccal cavities of infected as well as healthy subjects

\begin{tabular}{|c|c|c|}
\hline Consortium & $\begin{array}{l}\text { Source of } \\
\text { isolation }\end{array}$ & $\begin{array}{l}\text { Viable cell } \\
\text { count }(\mathrm{CFU} / \mathrm{ml})\end{array}$ \\
\hline$\overline{\text { DS1 }}$ & $\begin{array}{l}\text { Severe pit and fissure } \\
\text { caries from Subject } \mathrm{S} 1\end{array}$ & $2.7 \times 10^{7}$ \\
\hline DS2 & $\begin{array}{l}\text { Freshly initiated pit and } \\
\text { fissure caries from Subject S1 }\end{array}$ & $2.22 \times 10^{7}$ \\
\hline DS3 & $\begin{array}{l}\text { Non infected teeth } \\
\text { surface from Subject S1 }\end{array}$ & $4.92 \times 10^{5}$ \\
\hline DS4 & $\begin{array}{l}\text { Severe surface } \\
\text { caries from subject } \mathrm{S} 2\end{array}$ & $1.14 \times 10^{7}$ \\
\hline DS5 & $\begin{array}{l}\text { Severe surface caries with deformed } \\
\text { and discolored tooth from subject } S 3\end{array}$ & $6.6 \times 10^{2}$ \\
\hline DS6 & $\begin{array}{l}\text { Non infected teeth } \\
\text { surface from Subject S3 }\end{array}$ & $1.86 \times 10^{3}$ \\
\hline HS1 & Healthy subject 1 & $7.00 \times 10^{8}$ \\
\hline HS2 & Healthy subject 2 & $7.60 \times 10^{8}$ \\
\hline HS3 & Healthy subject 3 & $4.12 \times 10^{8}$ \\
\hline HS4 & Healthy subject 4 & $5.92 \times 10^{8}$ \\
\hline HS5 & Healthy subject 5 & $4.80 \times 10^{8}$ \\
\hline
\end{tabular}


OnLine J. Biol. Sci., 12 (2): 44-53, 2012

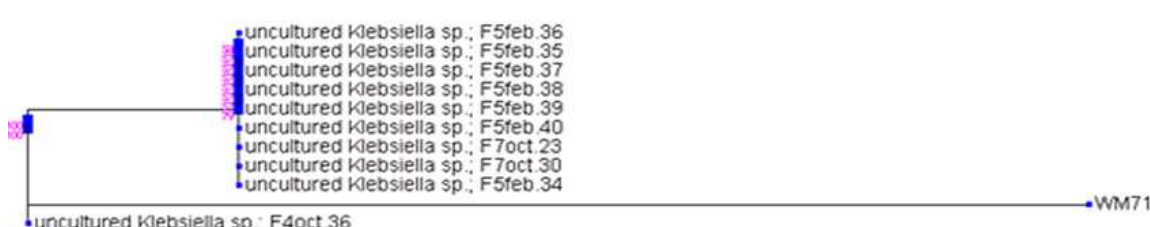

(a)

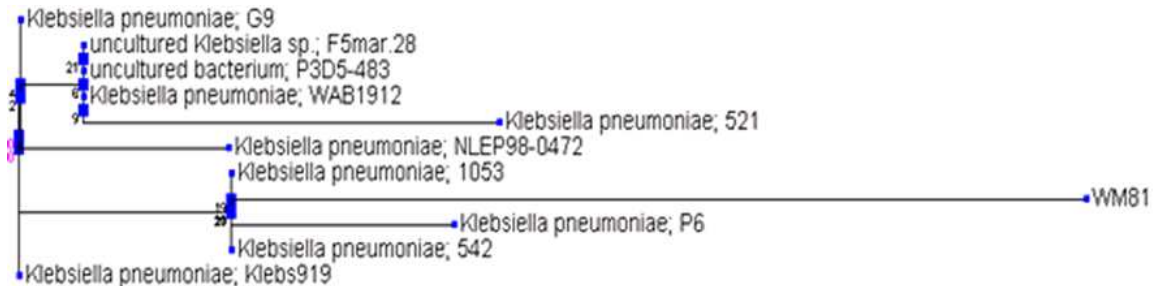

(b)

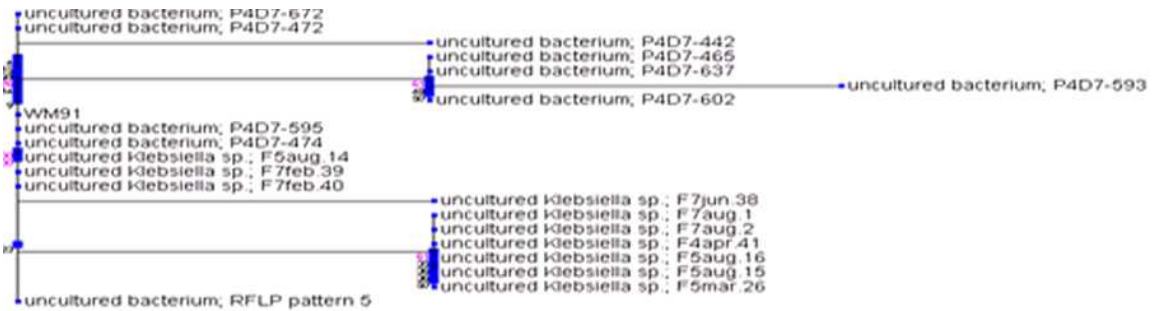

(c)

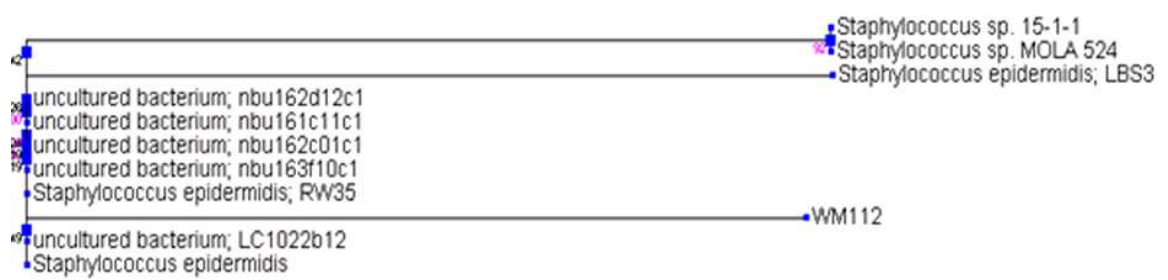

(d)

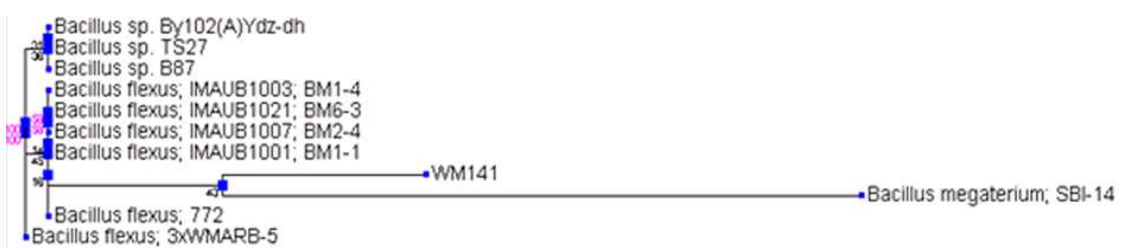

(e)

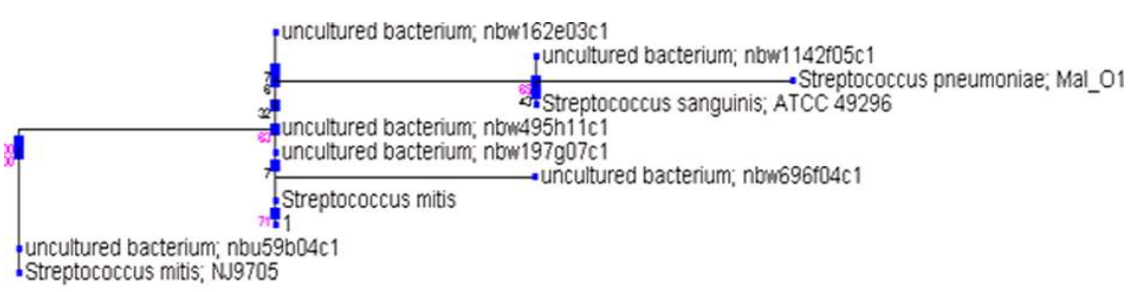

(f)

Fig. 2: Representative phylogenetic tree for each consortium constructed using neighbour joining method. The GenBank accession numbers are provided in Table 2. The different trees represent members from the following consortium: 2a. DS1, 2b. DS2, 2c. DS3, 2d. DS4, 2e. DS5, 2f. DS6. 
OnLine J. Biol. Sci., 12 (2): 44-53, 2012

Table 2: Table indicating the GenBank accession numbers of the novel 16S rRNA sequences and the statistical data representing the species richness (Shannon Diversity Index) and evenness (Equitability index) of microbial diversity in each of the consortium from infected subjects

\begin{tabular}{|c|c|c|c|}
\hline Consortium & $\begin{array}{l}\text { Gen bank } \\
\text { accession number }\end{array}$ & $\begin{array}{l}\text { Shannon } \\
\text { diversity index }\end{array}$ & $\begin{array}{l}\text { Equitibility } \\
\text { index }\end{array}$ \\
\hline$\overline{\text { DS1 }}$ & GU373986- GU374010 & 0 & Not defined \\
\hline DS2 & $\begin{array}{l}\text { GU374012, GU374014-15, GU374017, GU374020, } \\
\text { GU374023, GU374025, GU374027, GU374059, } \\
\text { GU374033, GU374038, GU374040, GU374042, } \\
\text { GU374044, GU374046-47, GU374049-50, GU374052-58 }\end{array}$ & 0.1679 & 0.2422 \\
\hline DS3 & $\begin{array}{l}\text { GU797253-56, GU797258-59, GU797261, } \\
\text { GU797263-67, HM195201-10 }\end{array}$ & 0.184 & 0.2655 \\
\hline DS4 & GU797268-82, HM195214-23 & 1.164 & 0.843 \\
\hline DS5 & GU797283-307 & 0.441 & 0.4016 \\
\hline DS6 & $\begin{array}{l}\text { GU374011, GU374013, GU374060, GU374016, } \\
\text { GU374018-19, GU374021-22, GU374024, } \\
\text { GU374026, GU374028-32, GU374034-37, GU374039, } \\
\text { GU374041, GU37403, GU374045, GU374048, GU374051 }\end{array}$ & 0.8385 & 0.7633 \\
\hline
\end{tabular}

Table 3: Table representing the antibiotic sensitivity profile of the different consortia both from healthy (HS1-HS5) and infected buccal cavities (DS1-DS6) as well as the three pure isolates (SRC_DSa, SRC_DSb and SRC_DSc). The abbreviation of the antibiotics

\begin{tabular}{|c|c|c|c|c|c|c|c|c|c|c|c|c|c|c|c|c|c|c|}
\hline Consortium & Cf5 & Mt4 & A 10 & T 30 & Ca 30 & Ro 30 & Do 30 & Va 30 & Cx 10 & $\operatorname{Tr} 30$ & Nx 10 & G 10 & R 15 & $\mathrm{~Pb} 100$ & $\mathrm{Cq} 30$ & N 30 & C 30 & Ctx30 \\
\hline HS1 & $\mathrm{S}$ & $\mathrm{R}$ & $\mathrm{R}$ & $\mathrm{S}$ & $S$ & $\mathrm{R}$ & I & $\mathrm{R}$ & $\mathrm{S}$ & $\mathrm{S}$ & $\mathrm{S}$ & $S$ & $\mathrm{R}$ & $\mathrm{R}$ & $\mathrm{S}$ & $\mathrm{S}$ & $S$ & $\mathrm{~S}$ \\
\hline HS2 & S & $\mathrm{R}$ & $\mathrm{R}$ & S & S & S & $\mathrm{R}$ & $\mathrm{R}$ & $\mathrm{R}$ & $S$ & $S$ & $S$ & $\mathrm{R}$ & $\mathrm{R}$ & S & I & $S$ & S \\
\hline HS3 & $S$ & $\mathrm{R}$ & $\mathrm{R}$ & $S$ & $S$ & $\mathrm{~S}$ & $S$ & $\mathrm{R}$ & $S$ & $S$ & $S$ & $S$ & $S$ & $\mathrm{R}$ & $\mathrm{R}$ & I & $S$ & $S$ \\
\hline HS4 & $S$ & $\mathrm{R}$ & $\mathrm{R}$ & $S$ & $S$ & $\mathrm{R}$ & $\mathrm{R}$ & $\mathrm{R}$ & $\mathrm{R}$ & $S$ & $S$ & $S$ & $\mathrm{R}$ & $S$ & $\mathrm{~S}$ & I & $S$ & $S$ \\
\hline HS5 & $S$ & $\mathrm{R}$ & $\mathrm{R}$ & S & S & $\mathrm{R}$ & S & $\mathrm{R}$ & $\mathrm{R}$ & $\mathrm{S}$ & $S$ & $\mathrm{~S}$ & $\mathrm{R}$ & $\mathrm{R}$ & S & $\mathrm{S}$ & $\mathrm{S}$ & S \\
\hline DS1 & I & $\mathrm{R}$ & $\mathrm{R}$ & $\mathrm{R}$ & S & $\mathrm{R}$ & $\mathrm{R}$ & $\mathrm{R}$ & $\mathrm{R}$ & S & S & S & $\mathrm{R}$ & S & I & I & S & S \\
\hline DS2 & $\mathrm{S}$ & $\mathrm{R}$ & $\mathrm{R}$ & I & S & $\mathrm{R}$ & I & $\mathrm{R}$ & $\mathrm{R}$ & $\mathrm{S}$ & $S$ & $\mathrm{~S}$ & $\mathrm{R}$ & S & I & $\mathrm{S}$ & $\mathrm{S}$ & S \\
\hline DS3 & S & $\mathrm{R}$ & $\mathrm{R}$ & $\mathrm{R}$ & S & $\mathrm{R}$ & S & $\mathrm{R}$ & $\mathrm{R}$ & $\mathrm{R}$ & $\mathrm{R}$ & $S$ & $\mathrm{R}$ & $\mathrm{S}$ & S & S & I & $\mathrm{R}$ \\
\hline DS4 & $S$ & S & S & S & S & S & S & S & I & $\mathrm{R}$ & S & S & S & $\mathrm{S}$ & S & S & S & S \\
\hline DS5 & $\mathrm{R}$ & $\mathrm{R}$ & $\mathrm{R}$ & $\mathrm{R}$ & $\mathrm{R}$ & $\mathrm{R}$ & $\mathrm{R}$ & $\mathrm{R}$ & $\mathrm{R}$ & S & $\mathrm{R}$ & $\mathrm{R}$ & $\mathrm{R}$ & S & $\mathrm{R}$ & $\mathrm{R}$ & $\mathrm{R}$ & $\mathrm{R}$ \\
\hline DS6 & $\mathrm{R}$ & $\mathrm{R}$ & $\mathrm{R}$ & $\mathrm{R}$ & $\mathrm{R}$ & $\mathrm{R}$ & $\mathrm{R}$ & $\mathrm{R}$ & $\mathrm{R}$ & $\mathrm{R}$ & $\mathrm{R}$ & $\mathrm{R}$ & $\mathrm{R}$ & $S$ & $\mathrm{R}$ & $\mathrm{R}$ & $\mathrm{R}$ & $\mathrm{R}$ \\
\hline \multicolumn{19}{|c|}{ Pure Isolates } \\
\hline $\begin{array}{l}\mathrm{SRC}_{-} \\
\mathrm{DSa}\end{array}$ & I & $\mathrm{R}$ & $\mathrm{R}$ & $\mathrm{S}$ & $\mathrm{R}$ & $\mathrm{R}$ & $\mathrm{R}$ & $\mathrm{R}$ & $\mathrm{R}$ & $\mathrm{S}$ & I & $\mathrm{R}$ & $\mathrm{R}$ & $\mathrm{S}$ & $\mathrm{R}$ & $\mathrm{R}$ & $\mathrm{S}$ & $\mathrm{R}$ \\
\hline $\begin{array}{l}\mathrm{SRC}_{-} \\
\mathrm{DSb}\end{array}$ & $\mathrm{S}$ & $\mathrm{R}$ & $\mathrm{R}$ & $\mathrm{S}$ & $\mathrm{R}$ & $\mathrm{S}$ & I & I & I & $\mathrm{S}$ & $\mathrm{S}$ & $\mathrm{R}$ & $\mathrm{S}$ & $\mathrm{R}$ & $\mathrm{S}$ & $\mathrm{R}$ & $\mathrm{S}$ & S \\
\hline $\begin{array}{l}\text { SRC_ } \\
\text { DSc }\end{array}$ & I & $\mathrm{R}$ & $\mathrm{R}$ & $\mathrm{R}$ & $\mathrm{R}$ & I & $\mathrm{R}$ & $\mathrm{R}$ & $\mathrm{R}$ & $\mathrm{S}$ & $\mathrm{S}$ & I & $\mathrm{R}$ & $\mathrm{S}$ & I & I & $\mathrm{S}$ & $\mathrm{R}$ \\
\hline
\end{tabular}

[Ampicillin(A), Cephadroxil (Cq), Chloramphenicol(C), Cloxacillin (Cx), Cephotaxime (Ce), Ceftazidime(Ca), Ciprofloxacin (Cf), Doxycycline Hydrochloride(Do), Gentamicin(G), Metronidazole (Mt), Neomycin(N), Norfloxacin (Nx), Polymyxin B(Pb), Rifampicin(R), Roxithromycin (Ro), Tetracycline(T), Trimethoprin (Tr), Vancomycin (Va), Cephotaxime(CTX),] with their respective concentration in mcg per disc is mentioned in the first row of the table. Here $\mathrm{R}$ denotes resistant, I denotes intermediate response and $\mathrm{S}$ denotes sensitive

Table 4: Results of antibiotic sensitivity assay conducted by using the rapid detection kit conducted for three consortia: one from healthy buccal cavity and two from infected subjects

\begin{tabular}{llll}
\hline Antibiotics & Normal & PIT caries & Surface caries \\
\hline $\mathrm{Nx}(10)$ & - & - & - \\
$\mathrm{Ca}(30)$ & - & - & + \\
$\mathrm{T} 30$ & - & - & - \\
$\mathrm{N} 30$ & + & - & - \\
Tr30 & - & - & - \\
Ro30 & - & - & - \\
G $(10)$ & - & - & - \\
R (30) & - & - & - \\
Ctx $(30)$ & - & - & - \\
A10 & - & + & + \\
Do30 & - & - & - \\
Pb 100 & + & + & + \\
Cq30 & - & - & - \\
Mt4 & + & + & - \\
C30 & - & - & - \\
Cf5 & - & - & - \\
Va30 & - & - & - \\
Cx30 & - & + & - \\
\hline
\end{tabular}

Detection of Plasmids in the isolated microbial consortia: Although all the 14 samples, five consortia from the healthy subjects, six consortia from the infected subjects and the three pure isolates, showed resistance to varied antibiotics, among them only four healthy samples (HS3, HS2, HS1, HS5) showed the presence of plasmid and so did the pure isolates SRC_DSa and SRC_DSc. Being transformed into an ampicillin sensitive host $E$ coli $\mathrm{DH} 5 \alpha$, these plasmids were found to confer the ampicillin resistance on them. Thus it can be inferred that the occurrence of the plasmid bearing ampicillin resistance gene is not linked to the incidence of dental caries, rather they can be present in certain groups of oral microbes.

Rapid antibiotic sensitivity detection kit: In order to enable antibiotic sensitivity detection in case of dental caries, the modified method of two step cultivation was followed. 
OnLine J. Biol. Sci., 12 (2): 44-53, 2012

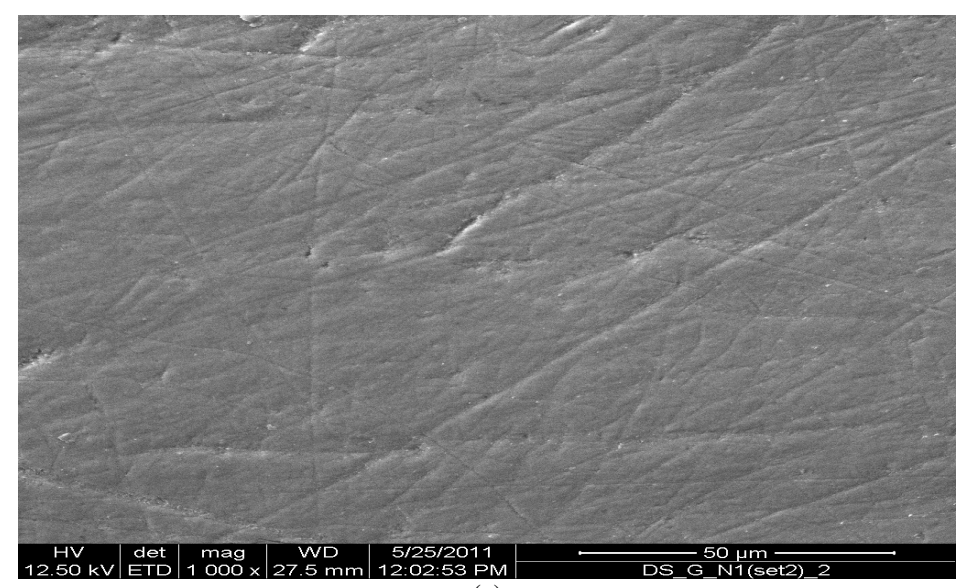

(a)

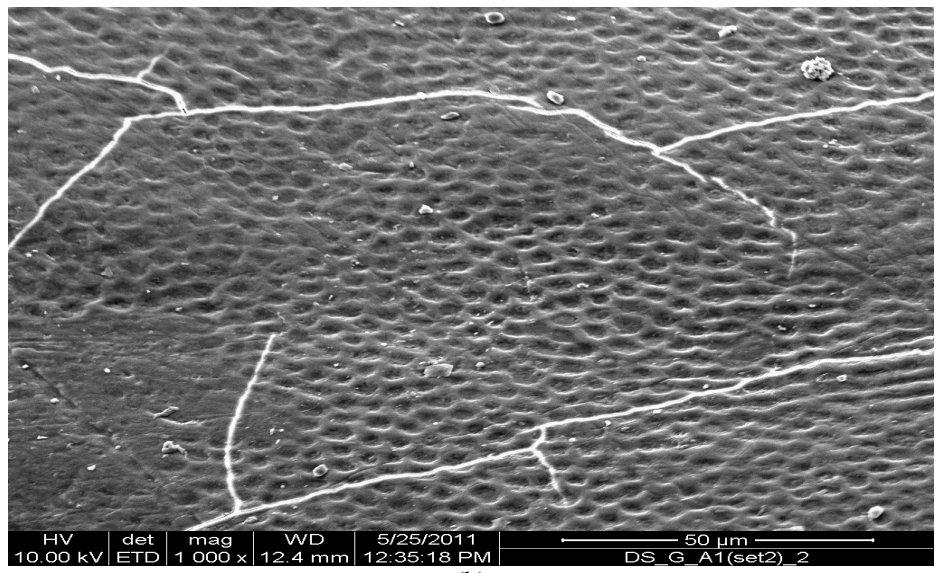

(b)

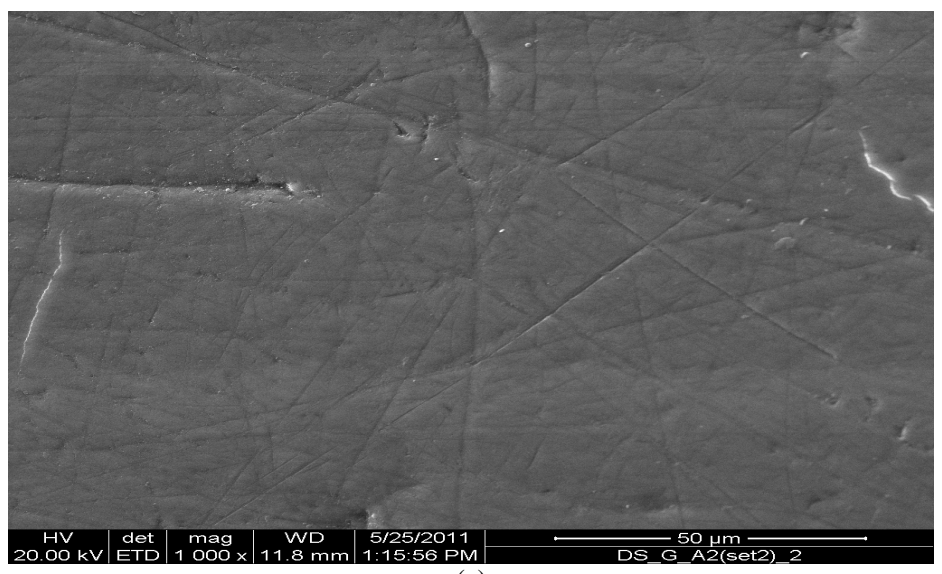

(c)

Fig. 3: Scanning Electron Micrograph of the surface of teeth. (a) Normal tooth of goat treated with simulated saliva and $1 \mathrm{M}$ sucrose solution for 21 days. (b) Surface of the bacteria infected goat tooth treated with simulated saliva and 1M sucrose solution for 21 days. (c) Surface of antibiotic treated bacteria infected tooth treated with simulated saliva and $1 \mathrm{M}$ sucrose solution for 21 days. Here antibiotic was applied everyday on the surface of the teeth for the entire period 
OnLine J. Biol. Sci., 12 (2): 44-53, 2012

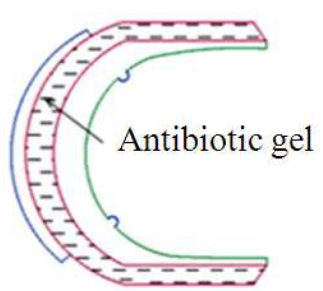

Top view

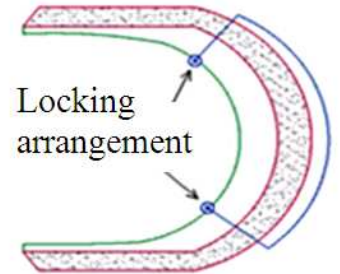

Bottom view

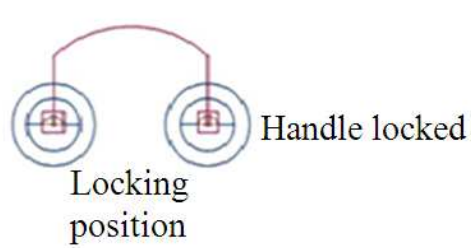

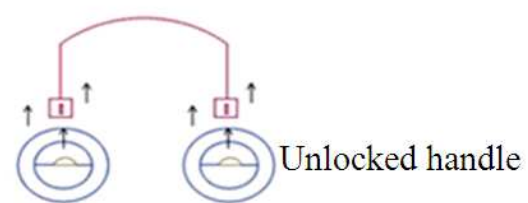

Open position

Fig. 4: Design of a device that would be made of moldable plastic for application of antibiotic to the teeth surface. It is fixed with a detachable handle and filled with antibiotic gel. This plastic would be placed around the teeth on the upper and lower jaws separately one by one. The plastic would take the shape of the teeth as in case of denture due to application of pressure. The handle would be released in each case by pulling it outwards after the casing is fixed tightly between the locked jaws. Thus the assembly with antibiotic gel would remain in contact with the surface of the teeth for $12 \mathrm{~h}$ (during night) minimizing the CFU on the teeth surface

The entire detection technique can be carried out in a small volume and the time duration can be scaled down to $18 \mathrm{~h}$ which is much less than the reported conventional procedure of 48-72 $\mathrm{h}$. The two step cultivation method ensures the uniform growth of the bacterial population before the antibiotic test (Fig. 1). Table 4 summarizes the assay results of three samples that were further selected, one from the healthy buccal cavity, the other two from infected subjects with the two different types of caries mentioned above. The extent of growth varies for each consortium but most of them showed resistance towards Ampicillin, Polymixin B, Roxithromycin and Metronidazole. The Result obtained using the two step cultivation and the conventional method were similar proving this method to be an alternative to the conventional method for antibiotic sensitivity testing.

Effect of antibiotic action on teeth surface: The effect of antibiotic action was checked on three sets of goat teeth. One was taken as control having neither bacterial nor antibiotic treatment, the second tooth set was treated with $S$ haemolyticus for studying the bacterial action on dentine surface and the third set was initially exposed to bacterial action followed by antibiotic treatment in the form of local application. The combination of antibiotic therapy was chosen in terms of Polymyxin B and Neomycin (Neosporin). The antibiotic effect on the teeth surface was determined by Scanning Electron Microscopy. As observed in Fig. 3ac, surface cracks and honey comb like structures were found in the second tooth set which was exposed exclusively to the bacterial infection without any further antibiotic application whereas no such surface alterations were seen in the third set which was treated with antibiotic gel post bacterial infection. The smooth tooth surface in the latter case was similar to the control tooth set.

Device for antibiotic application on the teeth: One of the effective ways of caries intervention is the local application of antimicrobials so as to reduce or eliminate plaque formation. The conventional approaches of using gels or toothpastes are not successful because of the inaccessibility of the agent to the entire surface of the affected dentine for a prolonged period of time. The proposed device is a simple model (Fig. 4). and has the advantage of being able to fit into the jaw of any individual. It can be used for regular use and ensures localized and prolonged application of the antibiotic gel.

\section{DISCUSSION}

The foremost objective of the study was to explore the cultivable microbiota associated with different types 
of dental caries seen in permanent teeth. Though existing reports suggest that a significant fraction of oral microbes is uncultivable, still the culture based approach would lead to isolation, identification and complete characterization of various pathogens causing dental caries in adult humans. The next objective was to obtain a comparative assessment of the microbial count between healthy and infected buccal cavities. As depicted in the result section, the viable cell count from the teeth surface of a healthy buccal cavity is much higher than the caries infected ones. This finding can be justified based on the Ecologic Plaque Hypothesis ( $\mathrm{Li}$ et al., 2005). In the primary stage of caries, the microbial invaders provide the compatible environment for the successive stages. But in the progressive stages, the microbial community diversity is drastically reduced because the latter species alters the environment to suppress the growth of the preliminary inhabitants. This theory can also explain the low Equitability Index and Shannon Diversity Index observed during the community analysis of the different consortia obtained from infected teeth. Moreover the culture based technique screens out only a fraction of the entire micro biota due to which the mean species richness and the Shannon Diversity is expected to be low. Thus, the ideal method to understand the diversity should be based on culture independent approach.

After the panel of microbes was screened, the next objective was to characterize the microbes by deciphering the molecular identity as well as studying the antibiotic sensitivity property. The antibiotic profile would help in prescribing the effective treatment method. The rapid antibiotic sensitivity detection kit designed during this study would ensure easy and faster diagnostics. The conventional approach of caries treatment was an oral or intravenous intake of antibiotics but since there is a very low blood supply to the dentin surfaces it inhibits the drug delivery at the lesion site. As a trial to develop an effective treatment method, a device was designed which can be loaded with antibiotic gel and then can be fixed on jaws to facilitate the localized application on the infected teeth surface. This device can be used in addition to the otherwise used methods of fluoride based toothpastes and gels.

\section{CONCLUSION}

To conclude, it can be stated that this study deals with understanding the microbial ecology of dental caries, deciphering the potent role of different species in caries development and thereafter development of novel diagnostic and treatment methods against this disease to slow down or stop demineralization of dentine.

\section{ACKNOWLEDGEMENT}

The authors would like to thank the Department of Biotechnology as well as Department of Atomic Energy for providing the fellowship; West Bengal University of Technology for providing the laboratory as well as computational facility. The authors also would like to thank Dr Dipak Mondal of Indian Veterinary Research Institute, Kolkata for his inputs during the in vitro dentine demineralization experiment.

\section{REFERENCES}

Aas, J.A., A.L. Griffen, S.R. Dardis, A.M. Lee and I. Olsen et al., 2008. Bacteria of dental caries in primary and permanent teeth in children and young adults. J. Clin. Microbiol., 46: 1407-1417. DOI: 10.1128/JCM.01410-07

Aas, J.A., B.J. Paster, L.N. Stokes, I. Olsen and F.E. Dewhirst, 2005. Defining the normal bacterial flora of the oral cavity. J. Clin. Microbiol., 43: 57215732. DOI: 10.1128/JCM.43.11.5721-5732.2005

Adarsh, V.K., M. Mishra, S. Chowdhury, M. Sudarshan and A.R. Thakur et al., 2007. Studies on metal microbe interaction of three bacterial isolates from east calcutta wetland. Online J. Biol. Sci., 7: 80-88. DOI: $10.3844 /$ ojbsci.2007.80.88

Islam, B., S.N. Khan and A.U. Khan, 2007. Dental caries: From infection to prevention. Med. Sci. Monit., 13: RA196-203. PMID: 17968308

Becker, M.R., B.J. Paster, E.J. Leys, M.L. Moeschberger and S.G. Kenyon et al., 2002. Molecular analysis of bacterial species associated with childhood caries. J. Clin. Microbiol., 40: 1001-1009. DOI: 10.1128/JCM.40.3.10011009.2002

Cate, J.M.T., 2009. The need for antibacterial approaches to improve caries control. Adv. Dent. Res., 21: 8-12. PMID: 19661048

Gafan, G.P., V.S. Lucas, G.J. Roberts, A. Petrie and M. Wilson, 2005. Statistical analyses of complex denaturing gradient gel electrophoresis profiles. J. Clin. Microbiol., 43: 3971-3978.

Ghosh, S., G. Roy and B. Mukherjee, 2009. Dental mold: A novel formulation to treat common dental disorders. AAPS Pharm. Sci. Tech., 10: 692-702. PMID: 19466555

Gross, E.L., E.J. Leys, S.R. Gasparovich, N.D. Firestone and J.A. Schwartzbaum et al., 2010. Bacterial 16S sequence analysis of severe caries in young permanent teeth. J. Clin. Microbiol., 48: 4121-4128. DOI: 10.1128/JCM.01232-10 
Hardie, J.M., 1992. Oral microbiology: current concepts in the microbiology of dental caries and periodontal disease. Br. Dent. J., 172: 271-278. PMID: 1576024

Houte, J.V., 1994. Role of micro-organisms in caries etiology. J. Dent. Res., 73: 672-681.

Li, Y., C.Y.S. Ku, J. Xu, D. Saxena and P.W. Caufield, 2005. Survey of oral microbial diversity using pcrbased denaturing gradient gel electrophoresis. J. Dent. Res., 84: 559-564. DOI: 10.1177/154405910508400614

Liljemark, W.F. and C. Bloomquist, 1996. Human oral microbial ecology and dental caries and periodontal diseases. Crit. Rev. Oral. Biol. Med., 7: 180-198. DOI: $10.1177 / 10454411960070020601$

Marsh, P.D., 2003. Are dental diseases examples of ecological catastrophes? Microbiology, 149: 279294. DOI: $10.1099 / \mathrm{mic} .0 .26082-0$

Nakano, K., H. Nemoto, R. Nomura, H. Inaba and H. Yoshioka et al., 2009. Detection of oral bacteria in cardiovascular specimens. Oral Microbiol. Immun., 24: 64-68. DOI: $10.1111 / \mathrm{j} .1399$ 302X.2008.00479.x

Nandy, P., A.R. Thakur and S.R. Chaudhuri, 2007. Characterization of bacterial strains isolated through microbial profiling of urine samples. Online J. Biol. Sci., 7: 44-51. DOI: 10.3844/ojbsci.2007.44.51

Nandy, P., S. Roy, A.R. Thakur and S.R. Chaudhuri, 2009. Comparative study on characterization of three Staphylococcal isolates from varied origin. J. Culture Collect., 6: 52-60.
Nasipuri, P., G.G. Pandit, A.R. Thakur and S.R. Chaudhuri, 2010. Comparative study of soluble sulphate reduction by bacterial consortia from varied regions of India. Am. J. Environ. Sci., 6: 152-158. DOI: 10.3844/ajessp.1010.152.158

Preza, D., I. Olsen, J.A. Aas, T. Willumsen and B. Grinde et al., 2008. Bacterial profiles of root caries in elderly patients. J. Clin. Microbiol., 46: 2015202. DOI: 10.1128/JCM.02411-07

Chaudhuri, S.R. and A.R. Thakur, 2006. Microbial genetic resource mapping of east calcutta wetlands. Curr. Sci., 91: 212-217.

Chaudhuri, S.R., A.R. Thakur, P. Nandy and S. Samanta, 2008. Urinary tract infection-a survey of local population. Am. J. Infect. Dis., 4: 117-123. DOI: 10.3844/ajidsp.2008.117.123

Ready, D., A.P. Roberts, J. Pratten, D.A. Spratt and M. Wilson et al., 2002. Composition and antibiotic resistance profile of microcosm dental plaques before and after exposure to tetracycline. J. Antimicrob. Chemother., 49: 769-775. DOI: 10.1093/jac/dkf005

Roberts, M.C., 1998. Antibiotic resistance in oral/respiratory bacteria. Crit. Rev. Oral. Biol. Med., $\quad 9$ : $522-540 . \quad$ DOI: $10.1177 / 10454411980090040801$

Srivastava, S.M. and M. Chaudhury, 2011. Evaluation of in vitro efficacy of cefepime sulbactam in combination. Eur. J. Dent Med., 3: 28-31.

Shivkumar, K.M., S.K. Vidya and G.N. Chandu, 2009. Dental caries vaccine. Indian J. Dent. Res., 20: 99106. 